\section{Annual Progress Report}

DE-FGO2-86ER53240
$D O E / E R / 53240--72$

DES2 040674

\title{
Theoretical and Numerical Studies in Magnetic Fusion
}

\section{Gyrokinetics and Transport Equations.}

The gyrokinetic equation has been employed to derive the transport theory for any topologicaly toroidal device possessing well defined magnetic surfaces $\psi(\mathbf{r}, t)=$ const.. Thus it includes the theory appropriate for a Tokamak with rotation as well as that for the Stellarator. The method does not require a detour via moment equations. It allows for finite mean flows. To illustrate the technique we apply it to the transport of density as follows.

Consider the gyrokinetic equation in conservation form

$$
\left.\frac{\partial}{\partial t}[\langle\langle\Delta\rangle\rangle f]\right)_{\text {roll }}=\frac{\partial}{\partial t}[\langle\langle\Delta\rangle\rangle f]+\nabla^{\prime} \cdot\left[\langle\langle\Delta\rangle\rangle \mathbf{v}^{n} f\right]+\frac{\partial}{\partial u^{\prime}}\left[\langle\langle\Delta\rangle\rangle\left\langle\left\langle u^{\prime}\right\rangle\right\rangle f\right]
$$

This is correct through first order in the formal small parameter $1 / \Omega$. The quantities entering the equation are defined as follows. Let the vectors $\mathbf{e}_{1}(r, t), \mathbf{e}_{2}(r, t), \mathbf{e}_{3}(r, t)=b(r, t)=B(r, t) / B(r, t)$ be a slowly varying right handed orthonormal set. Define $\mathbf{U}=\frac{(\mathbf{E}+\nabla \chi) \times \mathbf{B}}{B^{2}}$ where the potential $\chi$ is constructed so that $\mathbf{b} \cdot \nabla \chi=-\mathbf{b} \cdot \mathbf{E}$. Let $u=\mathbf{v} \cdot \mathbf{b}, \quad \mathbf{v}=u \mathbf{b}+\mathrm{U}, \mathbf{w}=\mathbf{v}-\mathrm{U}-u \mathbf{b}, \quad \mu=\frac{m w^{2}}{2 B}$, $\phi=\arctan \frac{\mathbf{e}_{2} \cdot \mathbf{w}}{\mathbf{e}_{1} \cdot \mathbf{w}}$, and $\mathbf{F}=\frac{1}{4}\left(\mathbf{e}_{1} \mathbf{e}_{1}-\mathbf{e}_{2} \mathbf{e}_{2}\right) \sin 2 \phi-\frac{1}{4}\left(\mathbf{e}_{1} \mathbf{e}_{2}+\mathbf{e}_{2} \mathbf{e}_{1}\right) \cos 2 \phi$. Then the Jacobian $\Delta=\frac{\partial(\mathbf{r}, \mathbf{v}, t)}{\partial\left(\mathbf{r}^{\prime}, \mu^{\prime}, t\right)}$, its average over $\phi^{\prime}$ is $\langle\langle\Delta\rangle\rangle=\left(1+\frac{\mathbf{b}^{\prime} \cdot \nabla \times \mathbf{V}^{\prime}}{\Omega^{\prime}}\right) \frac{B^{\prime}}{m}$ and $f=f\left(\mathbf{r}^{\prime}, \mu^{\prime}, u^{\prime}, t\right)$. The independent variables are

$$
\mathbf{r}^{\prime}=\mathbf{r}+\frac{(\mathbf{v}-\mathbf{U}) \times \mathbf{b}}{\Omega}
$$




$$
\begin{gathered}
\mu^{\prime}=\mu\left\{1-\frac{\mathbf{b} \cdot \nabla \times \mathbf{V}}{\Omega}-\frac{m \mathbf{w} \times \mathbf{b}}{\mu B \Omega} \cdot\left[\frac{\mu}{m} \nabla B+\left(\frac{\partial}{\partial t}+\mathbf{V} \cdot \nabla\right) \mathbf{V}+\frac{Z e}{m} \nabla \chi\right]-2 \mathbf{F}: \nabla \mathbf{V}\right\} \\
\phi^{\prime}=\phi+\frac{m \mathbf{w} \times \mathbf{b}}{2 \mu B \Omega} \cdot\left[\mathbf{b} \times\left(\frac{\partial}{\partial t}+\mathbf{V} \cdot \nabla\right) \mathbf{b}+\frac{2 \mu B}{m}\left(\nabla \mathbf{e}_{2}\right) \cdot \mathbf{e}_{1}\right]+\frac{(\mathbf{F} \times \mathbf{b}): \nabla \mathbf{V}}{\Omega} \\
u^{\prime}=u+\frac{\mu B}{m \Omega} \mathbf{b} \cdot \nabla \mathbf{b}+\frac{\mathbf{w} \times \mathbf{b}}{\Omega} \cdot\left[\left(\frac{\partial}{\partial t}+\mathbf{V} \cdot \nabla\right) \mathbf{b}+(\nabla \mathbf{b}) \cdot \mathbf{V}\right]+\frac{2 \mu B}{m \Omega} \mathbf{F}: \nabla \mathbf{b}
\end{gathered}
$$

To derive the surface averaged continuity equation we integrate (1) over all $\left\{\mu^{\prime}, \hat{\psi}^{\prime}, u^{\prime}\right\}$ space and in space over the volume $V^{\prime}$ interior of the moving topological torus $\psi^{\prime}=\psi(\mathbf{r}, t)=$ const $=\Psi$. The collision term integrates to zero since in the absence of chemical reactions collisions conserve number. There results since the integrals over the terms involving $\partial / \partial \mu^{\prime}$ and $\partial / \partial u^{\prime}$ yield zero

$$
\begin{aligned}
\int_{V^{\prime}} d^{3} r^{\prime} \int d \mu^{\prime} d \phi^{\prime} d u^{\prime} \frac{\partial}{\partial t}(\langle\langle\Delta\rangle\rangle f) & =-\int_{V^{\prime}} d^{3} r^{\prime} \nabla \cdot \int d \mu^{\prime} d \phi^{\prime} d u^{\prime}\langle\langle\Delta\rangle\rangle\left(\mathbf{V}^{\prime}+v_{d}^{\prime}\right) f \\
& =-\int_{V^{\prime}=} d^{2} r^{\prime} \int d \mu^{\prime} d \phi^{\prime} d u^{\prime}\langle\langle\Delta\rangle\rangle \mathbf{n}^{\prime} \cdot\left(\mathbf{V}^{\prime}+v_{d}^{\prime}\right) f
\end{aligned}
$$

But since the surface $\psi^{\prime}=\Psi$ is a magnetic surface the moving lines of force are tangent to it and since $\mathbf{n}=\frac{\nabla \psi}{|\nabla \psi|}$ is normal to it one has $\mathbf{n} \cdot \mathbf{b}=0$. Moreover $\mathbf{U}$ has been constructed so that $\mathbf{E}=-\nabla \chi-\frac{\mathbf{U} \times \mathbf{B}}{c}$ whence it follows from $c \nabla \times \mathbf{B}=-\frac{\partial \mathbf{B}}{\partial t}$ that $\frac{\partial \mathbf{B}}{\partial t}=\nabla(\mathbf{U} \times \mathbf{B})$. As is well known $\mathbf{U}$ can be interpreted as the velocity of the lines of force and $\frac{\partial \psi}{\partial t}+\mathbf{U} \cdot \nabla \psi=0$. Since in the transport case $\frac{1}{\Omega} \frac{\partial \ln \psi}{\partial t}$ is small we can to lowest significant order replace the primed quantities by their unprimed counterparts and (6) reduces to

$$
\int_{V} d^{3} r \int d \mu d \phi d u \frac{\partial}{\partial t}\left(\frac{B}{m} f\right)=-\int_{\psi=\psi} d^{2} r \int d \mu d \phi d u \frac{B}{m} \mathbf{n} \cdot\left(\mathbf{U}+\mathbf{v}_{d}\right) f
$$


But

$$
\begin{aligned}
\frac{d}{d t} \int_{V} d^{3} r \int d \mu d \phi d u \frac{B}{m} f= & \int_{V} d^{3} r \int d \mu d \phi d u \frac{\partial}{\partial t}\left(\frac{B}{m} f\right) \\
& +\int_{V} d^{3} r \int d \mu d \phi d u \frac{B}{m} f \mathbf{n} \cdot \mathbf{U}
\end{aligned}
$$

where the surface term represents the time rate of change in volume due to the motion of the surface. When (8) is employed in (7) there results

$$
\frac{d}{d t} \int_{v} d^{3} r \int d \mu d \phi d u \frac{B}{m} f=-\int_{\psi=\Psi} d^{2} r \int d \mu d \phi d u \frac{B}{m} \mathbf{n} \cdot v_{d} f
$$

Parallel results are obtained for all summational invariants like kinetic energy and angular momentum. All display $v_{d}$ as the effective velocity for transport.

\section{Boundary Conditions for Neo-Classical Transport}

An analysis is underway to determine the correct boundary conditions to apply to the transport equations. If there is no reflux from the boundaries it is clear that kinetically one must view as unpopulated all drift orbits that touch an absorber. This indicates that there is a boundary layer roughly an effectve drift orbit thickness in which the distribution function passes from almost Maxwellian to far from Maxwellian.

\section{Rough Estimation of the Neoclassical Transport Coefficients.}

A start has been made on the rough characterization and parametric dependence of the neo-classical transport transport coefficients. This consists in classifying the parts of energy-magnetic moment space that contribute and bounding the effective collision time that enters the expressions.

General Formulation of the Turbulence Problem. 
The general equations governing the ensemble average field quantities have been derived and are being analyzed. We will attempt to use experimental information to determine which terms are dominant and see if one can asymptotically match the associated fluxes in the turbulent outer layer to the neo-classical results which dominate the center.

The theory proceeds from the equations governing the center of mass plasma in conservation form.

mass

$$
\frac{\partial \rho}{\partial t}+\nabla \cdot(\rho \mathbf{v})=0
$$

momentum

$$
\frac{\partial}{\partial t}\left(\rho \mathbf{v}+\frac{\mathbf{E x B}}{4 \pi c}\right)+\nabla \cdot \Pi=0
$$

where

energy

$$
\Pi=\left[p+\frac{\mathbf{E}^{2}+\mathbf{B}^{2}}{8 p}\right] \mathbf{I}+\rho \mathbf{v v}-\frac{\mathbf{E E}+\mathbf{B B}}{8 \pi}
$$

where

$$
\begin{gathered}
U=\frac{1}{2} \rho v^{2}+\frac{p}{\gamma-1}+\frac{\mathbf{E}^{2}+\mathbf{B}^{2}}{8 \pi} \\
\mathbf{d}=\frac{1}{2} \rho v^{2} \mathbf{v}+\frac{\gamma p \mathbf{v}}{\gamma-1}+q+\left(\frac{c \mathbf{E x B}}{4 \pi}\right)
\end{gathered}
$$

We write for all field quantities $\xi=\xi^{\prime}+\xi^{\prime \prime}$, where the prime distinguishes the mean value and the double prime the fluctuation thereabout. Then (10),et seq. imply 


$$
\begin{aligned}
& \frac{\partial \rho^{\prime}}{\partial t}+\nabla \cdot\left(\rho^{\prime} v^{\prime}+<\rho^{\prime \prime} v^{\prime \prime}>\right)=0 \\
& \left.\frac{\partial}{\partial t}\left(\rho^{\prime} v^{\prime}+<\rho^{\prime \prime} v^{\prime \prime}\right\rangle+\frac{\mathbf{E}^{\prime} \times B^{\prime}}{4 \pi}+\left\langle\frac{\mathbf{E}^{\prime \prime} \times \mathbf{B}^{\prime \prime}}{4 \pi}\right)\right)+\nabla \cdot \Pi=0 \\
& \Gamma=\left[p^{\prime}+\frac{\mathbf{E}^{\prime 2}+\mathbf{B}^{\prime 2}}{8 \pi}\right] I+\rho^{\prime} v^{\prime} \mathbf{v}^{\prime}-\frac{\mathbf{E}^{\prime} \mathbf{E}^{\prime}+\mathbf{B}^{\prime} \mathbf{B}^{\prime}}{4 \pi} \\
& +\left\langle\frac{\mathbf{E}^{\prime \prime 2}+\mathbf{B}^{\prime \prime 2}}{8 \pi}\right\rangle \mathbf{I}+\left\langle\rho^{\prime} \mathbf{v}^{\prime \prime} \mathbf{v}^{\prime \prime}+\rho^{\prime \prime} v^{\prime} v^{\prime \prime}+\rho^{\prime \prime} v^{\prime \prime} v^{\prime}-\frac{\mathbf{E}^{\prime \prime} \mathbf{E}^{\prime \prime}+\mathbf{B}^{\prime \prime} \mathbf{B}^{\prime \prime}}{4 \pi}\right\rangle \\
& \frac{\partial U^{r}}{\partial t}+\nabla \cdot \mathbf{d}^{\prime} \\
& =Q+\left(\boldsymbol{J}-\Sigma \mathbf{E}^{\prime \prime}\right) \cdot\left(\mathbf{E}^{\prime}+\frac{\mathbf{v}^{\prime} \mathbf{x} \mathbf{B}^{\prime}}{c}\right)+\left\langle\left(\mathbf{J}^{\prime \prime}-\Sigma \mathbf{E}^{\prime \prime}-\Sigma^{\prime \prime} \mathbf{F}^{\prime \prime}\right) \cdot\left(\mathbf{E}^{\prime \prime}+\frac{\mathbf{v}^{\prime \prime} \mathbf{x} \mathbf{B}^{\prime}}{c}+\frac{\mathbf{v}^{\prime} \mathbf{x} \mathbf{B}^{\prime \prime}}{c}\right)\right\rangle \\
& \mathbf{d}^{\prime}=\frac{\gamma}{\gamma-1}\left(p^{\prime} \mathbf{v}^{\prime}+\left\langle p^{\prime \prime} \mathbf{v}^{\prime \prime}\right\rangle\right)+\mathbf{q}^{\prime} \\
& +\frac{c}{4 \pi}\left(\mathbf{E}^{\prime} \mathbf{E}^{\prime}+\left\langle\mathbf{E}^{\prime \prime} \mathbf{B}^{\prime \prime}\right\rangle\right) \\
& \left.+\frac{1}{2}\left(\rho^{\prime} v^{\prime}+\left\langle r^{\prime \prime} v^{\prime \prime}\right\rangle\right)\left(v^{\prime 2}+\left\langle v^{\prime \prime 2}\right\rangle\right)+\frac{1}{2}\left\langle\left(v^{\prime 2}+<v^{\prime \prime 2}\right\rangle\right)\right\rangle \\
& \left.+\left\langle\left(\rho^{\prime \prime} v^{\prime \prime}-<\rho^{\prime \prime} v^{\prime \prime}\right\rangle+\rho^{\prime} v^{\prime \prime}+\rho^{\prime \prime} v^{\prime}\right)\left(v^{\prime \prime 2}-<v^{\prime \prime 2}>+2 v^{\prime} v^{\prime \prime}\right)\right\rangle
\end{aligned}
$$

Equations for the fluctuating quantities can be gotten by subtracting the average equation from its original counterpart. Conservation of mass yields

$$
\frac{\partial \rho^{\prime \prime}}{\partial \imath}+\nabla \cdot\left(\rho^{\prime \prime} v^{\prime}+\rho^{\prime} v^{\prime \prime}+\rho^{\prime \prime} v^{\prime \prime}-\left\langle\rho^{\prime \prime} v^{\prime \prime}\right\rangle\right)=0
$$




$$
\begin{gathered}
\frac{\partial}{\partial t}\left[\rho^{\prime} \mathbf{v}^{\prime \prime}+\rho^{\prime \prime} v^{\prime}+\frac{\mathbf{E}^{\prime} \mathbf{x} \mathbf{B}^{\prime \prime}}{4 \pi c}+\frac{\mathbf{E}^{\prime \prime} \mathbf{x} \mathbf{B}^{\prime}}{4 \pi c}\right]+\nabla \cdot \Gamma^{\prime}=0 \\
\Pi^{\prime \prime}=\left[p^{\prime \prime}+\frac{\mathbf{E}^{\prime} \cdot \mathbf{E}^{\prime \prime}+\mathbf{B}^{\prime} \cdot \mathbf{B}^{\prime \prime}}{4 \pi}\right] \mathbf{I}+\rho^{\prime}\left[\mathbf{v}^{\prime} \mathbf{v}^{\prime \prime}+\mathbf{v}^{\prime \prime} v^{\prime}\right] \\
+\rho^{\prime \prime} \mathbf{v}^{\prime} \mathbf{v}^{\prime}-\frac{\mathbf{E}^{\prime} \mathbf{E}^{\prime \prime}+\mathbf{B}^{\prime} \mathbf{B}^{\prime \prime}+\mathbf{E}^{\prime \prime} \mathbf{E}^{\prime}+\mathbf{B}^{\prime \prime} \mathbf{B}^{\prime}}{4 \pi}
\end{gathered}
$$

etcetera. 

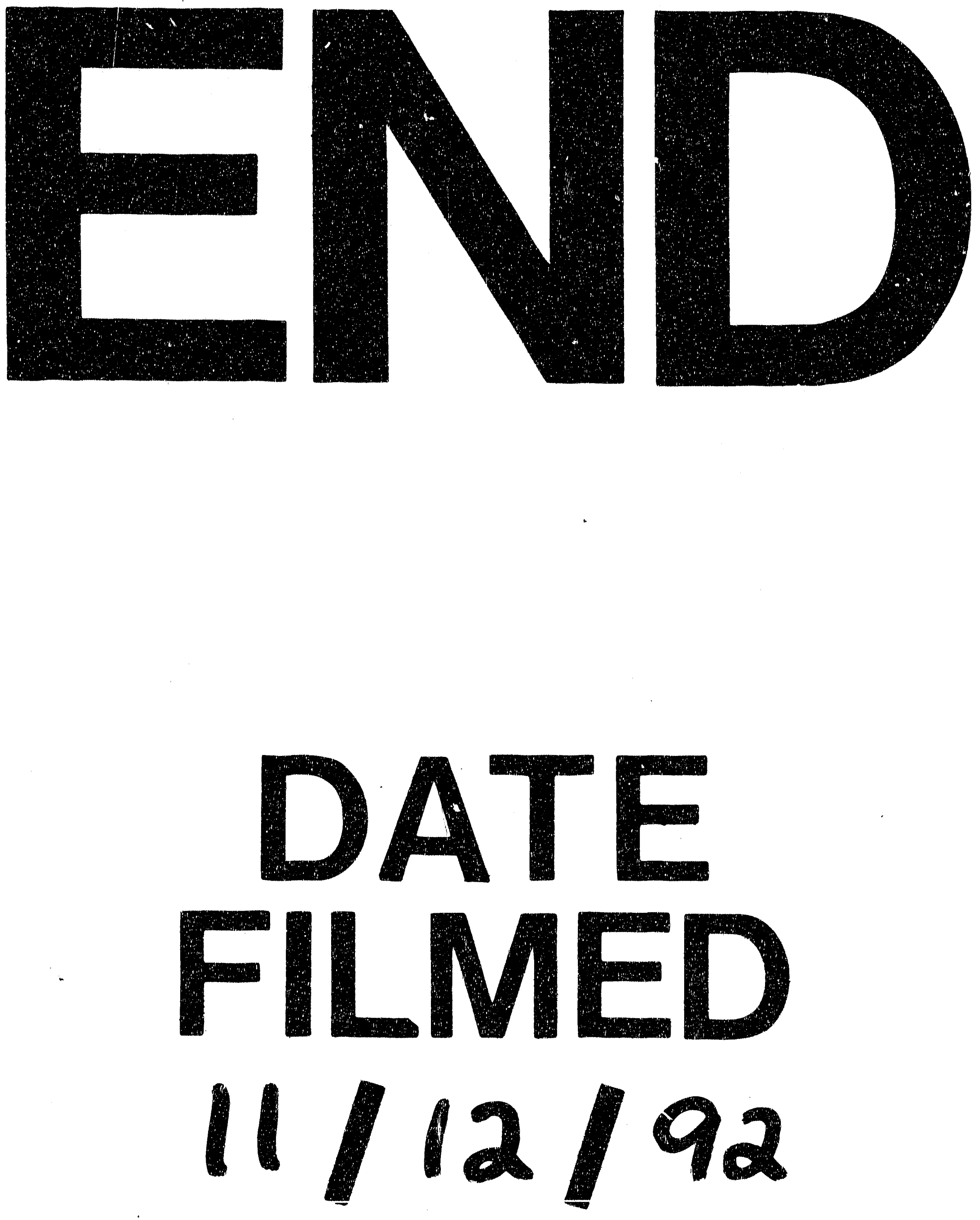

1 
